\title{
Metagenomic analysis of antibiotic resistance genes in coastal industrial mariculture systems
}

\author{
Jian-Hua Wang ${ }^{\mathrm{a}}$, Jian $\mathrm{Lu}^{\mathrm{a}, *}$, Yu-Xuan Zhang ${ }^{\mathrm{a}}$, Jun $\mathrm{Wu}^{\mathrm{b}}$, Yongming Luo ${ }^{\mathrm{a}}$, Hao Liu ${ }^{\mathrm{c}}$ \\ ${ }^{a}$ Key Laboratory of Coastal Environmental Processes and Ecological Remediation, Yantai Institute of Coastal Zone Research, Chinese Academy of Sciences, Yantai, \\ Shandong 264003, People's Republic of China \\ ${ }^{\mathrm{b}}$ Qinghai Institute of Salt Lakes, Chinese Academy of Sciences, Xining, Qinghai 810008, People's Republic of China \\ ' Shandong Oriental Ocean Sci-tech Co. Ltd, Yantai, Shandong 264003, People's Republic of China
}

G R A P H I C A L A B S T R A C T

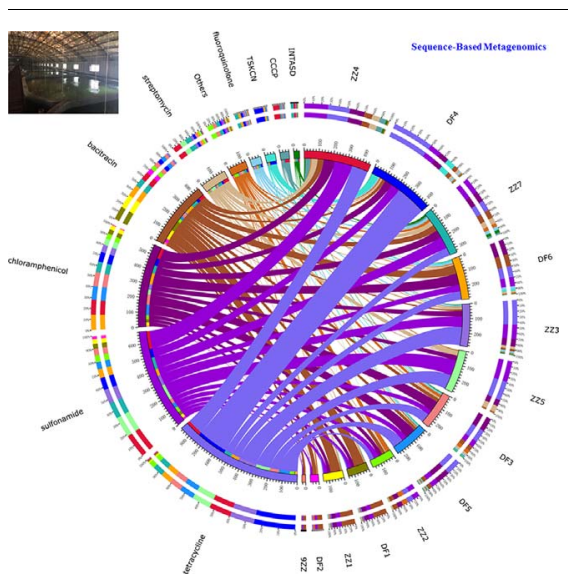

\section{A R T I C L E I N F O}

\section{Keywords:}

Antibiotic resistance genes

Metagenomics

Conventional mariculture system

Recirculating mariculture system

Coastal zone

\begin{abstract}
A B S T R A C T
The overuse of antibiotics has posed a propagation of antibiotic resistance genes (ARGs) in aquaculture systems. This study firstly explored the ARGs profiles of the typical mariculture farms including conventional and recirculating systems using metagenomics approach. Fifty ARGs subtypes belonging to 21 ARGs types were identified, showing the wide-spectrum profiles of ARGs in the coastal industrial mariculture systems. ARGs with multiple antibiotics resistance have emerged in the mariculure systems. The co-occurrence pattern between ARGs and microbial taxa showed that Proteobacteria and Bacteroidetes were potential dominant hosts of ARGs in the industrial mariculture systems. Typical nitrifying bacteria such as Nitrospinae in mariculture systems also carried with some resistance genes. Relative abundance of ARGs in fish ponds and wastewater treatment units was relatively high. The investigation showed that industrial mariculture systems were important ARGs reservoirs in coastal area, indicating the critical role of recirculating systems in the terms of ARGs pollution control.
\end{abstract}

\footnotetext{
* Corresponding author.

E-mail address: jlu@yic.ac.cn (J. Lu).
} 


\section{Introduction}

Playing a major role in both human and animal husbandry, antibiotics are extensively used for therapeutic treatment and growth promoters in aquaculture and farming (Xu et al., 2015). Long-term overuse of antibiotics has posed a propagation of antibiotic resistance genes (ARGs) in the environment, associating with the acquisition of drug resistance (Abriouel et al., 2008). ARGs were indicated as emerging contaminants by Pruden et al. (2006) and played an important role in antibiotic resistance. Increasing attention has been paid to environmental spread of ARGs due to their potential implications for human health and ecological environment. Aquaculture farms have been proved as reservoirs for ARGs in the environment due to the abuse of antibiotics (Miranda et al., 2013), thus attracting more attention on ARGs in aquaculture environment due to the rapid development of global aquaculture industry. The conventional (without recirculation unit) and recirculating aquaculture systems are two typical integrated aquaculture-agriculture systems. In these two farming systems, the animal manure and urine are continuously discharged directly into culture ponds. In the recirculation system, the aquaculture wastewater is circulated and reused after a systemic treatment while the aquaculture wastewater in the conventional system without recirculation is discharged into the sea directly or with simple treatment (Su et al., 2011; Xiong et al., 2015). Therefore, the aquaculture systems lead to the spread of ARGs and antibiotic resistance bacteria (Muziasari et al., 2017; Xiong et al., 2015).

Culture-independent molecular approaches have provided powerful tools to explore ARGs in nature and address the dissemination of ARGs in different environments (Yang et al., 2014; Zhu et al., 2017). Quantitative PCR (qPCR) and metagenomics analysis provide a comprehensive and quantitative insight on ARGs in nature (Aydin et al., 2016; Guo et al., 2017; Li et al., 2015; Muziasari et al., 2017).

Zhu et al. (2017) had profiled ARGs in sediments from 18 estuaries over $4000 \mathrm{~km}$ coastlines of China using high-throughput quantitative PCR. They found that ARGs in coastal samples were diverse and abundant, with over 200 different resistance genes being detected. It has been confirmed that mariculture farms are responsible for the enrichment of ARGs in coastal sediments using high-throughput quantitative PCR (Muziasari et al., 2017). Although qPCR including highthroughput qPCR is sensitive in detection of ARGs, it relies on specific primers designed from known target sequences so as not to detect unknown ARGs. Metagenomics approach is a high-throughput sequencing method which can provide information of the prevalence and diversity of ARGs, thus enabling discovery of novel ARGs by functional screening of metagenomics libraries (Guo et al., 2017; Hu et al., 2013; Yang et al., 2014). Metagenomics approach has been used to characterize the distribution of ARGs in various environments including human gut, wastewater, river water, drinking water, and soil (Guo et al., 2017; Hu et al., 2013; Li et al., 2015; Xiao et al., 2016). Rare report is available on the investigation of ARGs in coastal industrial mariculture systems using metagenomics analysis techniques.

Antibiotics are widely applied in coastal mariculture farms to prevent diseases and promote fish growth used as feed additives. Being a major aquaculture-agriculture country of world, China has the world largest scale of coastal mariculture industry and accounts for about 71\% of the total global aquaculture production (Sapkota et al., 2008). The antibiotics abuse in aquaculture farms require scientific evaluation of their impacts on ARGs in the environment (Gao et al., 2012; Miranda et al., 2013; Muziasari et al., 2017; Xiong et al., 2015). It is necessary to study the ARGs distribution in the industrial aquaculture systems with or without recirculation unit because they are the most popular farming systems used in coastal areas. The objectives of the present study are to obtain initial information on the profiles of ARGs in coastal industrial mariculture systems using metagenomics analysis techniques.

\section{Materials and methods}

\subsection{Sample collection}

The water samples were collected from two industrial mariculture systems with or without recirculation unit in Yantai City which is the most important mariculture city in China. The production of seawater industrial farming of Yantai City was 28,000 tons in 2015, accounting for $1 / 6$ of China. The mariculture systems included the conventional industrial mariculture system (ZZ farm) without recirculation unit and the recirculating mariculture system (DF farm). The water sample collected from each sampling point of the conventional system (ZZ farm) was marked as ZZ1, ZZ2, ZZ3, ZZ4, ZZ5, and ZZ6, respectively. The samples ZZ1, ZZ6, and ZZ7 refer to the influent, the stabilization and coastal water sample, respectively, while ZZ2, ZZ3, ZZ4, and ZZ5 refer to the sample of fish Ponds 1, 2, 3, and 4, respectively. The Pond 1 in ZZ farm is for Penaeus vannamei, Pond 2 is for Scophthalmus maximus, Pond 3 is for fish-fry of S. maximus, and Pond 4 is for Platichthys stellatus. The water samples collected from each sampling point of the recirculating system (DF farm) were marked as DF1, DF2, DF3, DF4, DF5, and DF6, respectively. The samples DF1, DF2, DF3, DF4, DF5, and DF6 refer to the influent, fish pond, protein separator, biochemical pool for wastewater treatment, biological aerobic pool for wastewater advanced treatment, and the recycled water. The fish pond in DF farm is for Atlantic salmon. Each water sample (30 L/point) was filtered through $0.22 \mu \mathrm{m}$ micropore membrane and kept in $-86^{\circ} \mathrm{C}$ for further study.

\subsection{DNA extraction, library preparation and sequencing}

The filtered samples were transported on dry-ice to Novegene (Beijing, China) for DNA extraction and sequencing. The total genomic DNA was extracted by CTAB/SDS method, and DNA degradation degree and potential contamination was monitored on $1 \%$ agarose gels. DNA purity (OD260/OD280, OD260/OD230) was checked using the NanoPhotometer ${ }^{\circledR}$ spectrophotometer (IMPLEN, CA, USA). DNA concentration was measured using QubitA ${ }^{\circledR}$ dsDNA Assay Kit in Qubit $^{\circledR} 2.0$ Flurometer (Life Technologies, CA, USA). OD values ranged from 1.8 to 2.0. DNA contents above $1 \mu \mathrm{g}$ were used to construct library.

Sequencing libraries were generated using NEBNext ${ }^{\circledR}$ Ultra $^{\mathrm{TM}}$ DNA Library Prep Kit for Illumina (NEB, USA) following manufacturer's recommendations and index codes were added to attribute sequences to each sample. Briefly, the DNA sample was fragmented by sonication to a size of $350 \mathrm{bp}$, then DNA fragments were end-polished, A-tailed, and ligated with the full-length adaptor for Illumina sequencing with further PCR amplification. At last, PCR products were purified (AMPure XP system) and libraries were analyzed for size distribution by Agilent2100 Bioanalyzer and quantified using real-time PCR.

The clustering of the index-coded samples was performed on a cBot Cluster Generation System according to the manufacturer's instructions. After cluster generation, the library preparations were sequenced on an Illumina HiSeq platform and paired-end reads were generated. The metagenomic data sets were filtered using a Readfq (V8, http://github. $\mathrm{com} / \mathrm{cjfields} / \mathrm{readfq}$ ) to remove raw reads that: included more than 40 nucleotides with quality value lower than 38 , had more than $15 \mathrm{bp}$ overlap with adapter sequences, and included $\mathrm{N}$ base exceeding $10 \mathrm{bp}$.

After quality filtering, clear reads were assembled into scaftigs by MEGAHIT assembler (V1.04- $\beta$, set as-presets meta-large (-min-count 2k-min 27-k-max 87-k-step 10)) (Qin et al., 2014) and interruption from the $\mathrm{N}$ joints (Qin et al., 2014). The clean data were mapped against scaftigs by SoapAligner (soap2.21, set as identity $\geq 90 \%$, -m 200, -x 400). The unused reads from each sample were assembled using MEGAHIT and set as the same parameters. Only scaftigs longer than $500 \mathrm{bp}$ were used for further analysis. The successfully aligned reads were assigned as "assembled reads". The bioinformatic analysis on the community structure and the abundance of ARGs was carried out as follows. The sequences described in this study were deposited in 
European Nucleotide Archive with accession numbers (ERP024470). Overall, Illumina sequencing generated more than $10 \mathrm{~Gb}$ of reads for each sample. After quality filtering, each sample still contained more than $10 \mathrm{~Gb}$ high-quality sequence reads, which were then assembled using MEGAHIT assembly algorithm. Totally, 7,394,585 scaftigs longer than $500 \mathrm{bp}$ were assembled from the metagenomic reads of the 13 samples. The minimum scaftig length of $500 \mathrm{bp}$ was chosen in order to ensure a reasonable length for open reading frames (ORF) prediction and obtain a dataset of manageable size. The percentage of reads that could be assembled into scaftigs over 500 bp was $5.64 \%$ for the 13 samples.

\subsection{Gene prediction from scaftigs}

ORFs (minimum length of 100 nucleotides) were predicted on scaftigs longer than $500 \mathrm{bp}$ using MetaGeneMark (V2.10, default setting). Then, a non-redundant gene catalogue was constructed with CDHIT (V4.5.8, set as -c 0.95, -G 0, -aS 0.9, -g 1, -d 0). To determine the abundance of genes, reads were realigned to the gene catalogue with SoapAligner (set as set as identity $\geq 95 \%$, -m 200, -x 400). Only genes with $\geq 2$ mapped reads were deemed to be Unigenes for further analysis (Li et al., 2014). The abundance of genes was calculated by counting the number of reads and normalizing by gene length (Karlsson et al., 2012; Villar et al., 2015).

\subsection{Taxonomic classification}

The Unigenes for each sample were searched against the NCBI NR database (V20161115) using DIAMOND (V 0.7.9, set as blastp, -e 1e-5) (Buchfink et al., 2015) with an E-value $<1 \times 10^{-20}$. The taxonomical level of each gene was determined by the lowest common ancestorbased algorithm and implemented in MEGAN (Karlsson et al., 2012). The abundance of a taxonomic group was calculated by summing the abundance of genes annotated to a feature.

\subsection{ARGs analysis}

The Unigenes were searched for ARGs against the widely accepted ARGs database ARDB using DIAMOND (V 0.7.9, set as blastp, -e 1e-5). A read was annotated as an ARG-like fragment if its best BLASTx hit in clean ARDB had a sequence similarity above $90 \%$ and an alignment length over 25 amino acids (Tian et al., 2016; Kristiansson et al., 2011). The proportions of different types of ARG-like sequences in 'total ARGlike sequences' and 'total metagenome sequences' were defined as 'percentage' (\%) and 'abundance' (ppm, 1 read in 1 million reads), respectively (Xiao et al., 2016; Yang et al., 2013).

\section{Results and discussion}

\subsection{Occurrence of ARGs types in coastal industrial mariculture systems}

To evaluate the mariculture farms impacts on the surrounding coastal marine environment, metagenomics approach was firstly used for investigating the ARGs profiles of coastal industrial maricultures farms. Through high-throughput sequencing-based metagenomics analysis with structured database of ARDB, 50 ARGs subtypes belonging to 21 ARGs types were identified in the samples from the industrial mariculture systems (Fig. 1). The detected ARGs included genes conferring resistance to antibiotics including tetracycline, sulfonamide, chloramphenicol, bacitracin, tigecycline, streptomycin, kanamycin, cephamycin, cephalosporin, carbapenem, penicillin, lincosamide, streptogramin, macrolide, fluoroquinolone, carbenicillin, isepamicin, netilmicin, tobramycin, amikacin, sisomicin, erythromycin, spectinomycin, dibekacin, gentamicin, bleomycin, trimethoprim, paromomycin, neomycin, lividomycin, ribostamycin, glycylcycline, and roxithromycin. The high detection frequency (90\%-100\%) of ARGs conferring resistance to tetracycline, chloramphenicol, streptomycin, sulfonamide, and fluoroquinolone suggested the wide application of these common antibiotics in maricultue systems. Wide-spectrum profiles of ARGs in the other environments were also observed using metagenomic approach. More than 200 different resistance genes have been detected in the estuarine sediment of China (Zhu et al., 2017). Li et al. (2015) found 260 ARGs subtypes belonging to 18 types by investigating the wide-spectrum profiles of ARGs in 50 samples from 10 typical environments using metagenomic approach. Xiao et al. (2016) found 110 subtypes ARGs belongings to 16 types ARGs in paddy soils. Fang et al. (2015) performed metagenomic survey on the ARGs and pathogens in long-term greenhouse soils to obtain 22 classes of ARGs and 46 species of human pathogenic bacteria carrying ARGs.

ARGs with multiple antibiotics resistance have emerged in the mariculure systems (Fig. 1). ARGs with multiple antibiotics resistance to tigecycline-streptomycin-kanamycin-ciprofloxacin-norfloxacin (TSKCN), cephamycin-cephalosporin-carbapenem-penicillin (CCCP), lincosamidestreptogramin-macrolide (LSM), carbenicillin-penicillin (CP), isepamicinnetilmicin-tobramycin-amikacin-sisomicin-dibekacin (INTASD), spectinomycin-streptomycin (SS), tobramycin-kanamycin-sisomicin-dibekacin-gentamicin (TKSDG), chloramphenicol-fluoroquinolone (CF), paromomycinneomycin-kanamycin-lividomycin-ribostamycin-gentamincin (PNKLRG), netilmicin-tobramycin-sisomicin-dibekacin-gentamicin (NTSDG), and glycylcycline-fluoroquinolone-roxithromycin-erythromycin (GFRE) have been detected. The distribution of ARGs types detected in each sample by Circos (Krzywinski et al., 2009) was shown in Fig. 2. ARGs conferring multiple resistance to three antibiotics lincosamide, streptogramin and macrolide (LSM) appeared in fish pond of $P$. vannamei (sample ZZ2). ARGs conferring multiple resistance to five antibiotics tigecycline, streptomycin, kanamycin, ciprofloxacin and norfloxacin (TSKCN) were found in the biochemical pool for water treatment (sample DF4). The results indicated the mariculture farms were crucial reservoir of multi-resistance genes. A lot of multi-resistance genes or bacteria have been found in aquaculture environment. Su et al. (2011) isolated 77 Enterobacteriaceae strains with multi-resistance to seven antibiotics from integrated fish farms in south China. According to Gao et al. (2012), at least two of the genes, tetM, tetO, tetT, tetW, sul1 and sul2 were detected in Bacillus spp. and Acinetobacter lwofii, and all of the above genes were detected in B. cereus, suggesting the occurrence of multiresistance in aquaculture environment.

\subsection{Abundance of ARGs types in coastal industrial mariculture systems}

The relative abundance of ARGs in different samples was shown in Fig. 3. The relative abundance of total ARGs in the two farms in this study were about $0.27-4.55 \times 10^{-4} \mathrm{ppm}$ (Fig. 3a). The high relative abundance of ARGs conferring resistance to tetracycline, sulfonamide, and chloramphenicol suggested the intensive application of these common antibiotics in maricultue systems. The high relative abundance of ARGs subtype (Fig. 4) conferring resistance to tetracycline, sulfonamide, and chloramphenicol confirmed the intensive usage of these three kinds of antibiotics in coastal industrial mariculture systems. The relative abundances of ARGs in fish ponds (ZZ2, ZZ3, ZZ4 and ZZ5) were much higher than those of the influent (ZZ1) and effluent (ZZ6) samples, suggesting that the fish ponds are the main hotpot of ARGs. According to the relative abundance of ARGs and distribution of ARG types (in relation to antibiotic types) detected in different samples, ARGs were widespread in the water samples at each stage of the two farms. Additionally, the ARGs relating to tetracycline, sulfonamide, chloramphenicol or bacitracin were the most abundant and commonly distributed ARGs in these samples, with the average percentages of the 4 types ARGs in each sample exceeding 15\% (Fig. 3b). The investigation indicated that coastal industrial mariculture systems were potential ARGs reservoirs for coastal waters. The wastewater treatment plants (WWTPs), rivers, drinking water, feces and wastewater from livestock farm, and manured soils have been considered as important reservoirs for ARGs (Yang et al., 2013; Li et al., 2015; Fang et al., 2015). The 


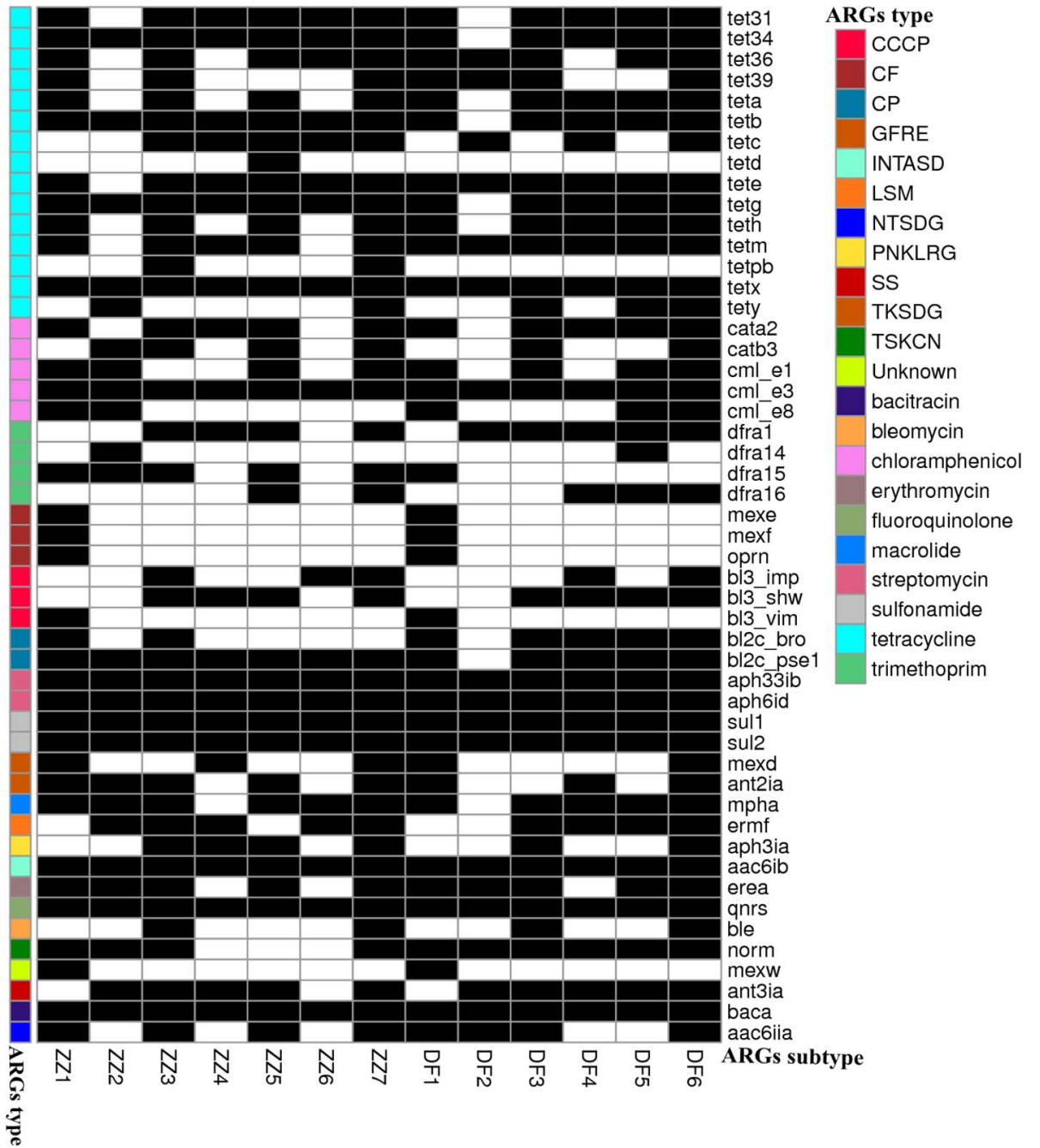

Fig. 1. Heat map of ARGs detection in coastal industrial mariculture systems. Black refers to positive while white refers to negative detection. The genes that can resist two or more antibiotics are described in acronyms: tigecyclinestreptomycin-kanamycin-ciprofloxacin-norfloxacin

(TSKCN), cephamycin-cephalosporin-carbapenem-penicillin (CCCP), lincosamide-streptogramin-macrolide (LSM), carbenicillin-penicillin (CP), isepamicin-netilmicin-tobramycin-amikacin-sisomicin-dibekacin ((INTASD), spectinomycin-streptomycin (SS), tobramycin-kanamycin-sisomicin-dibekacin-gentamicin (TKSDG), chloramphenicolfluoroquinolone (CF), paromomycin-neomycin-kanamycinlividomycin-ribostamycin-gentamincin (NTSDG), glycylcycline-fluoroquinolone-roxithromycin-erythromycin (GFRE). In the conventional mariculture system (ZZ farm), samples $Z Z 1, Z Z 6$, and ZZ7 refer to the influent, the stabilization and coastal water sample, respectively while ZZ2, ZZ3, ZZ4, and ZZ5 refer to the sample of fish pond 1, 2, 3, and 4, respectively. In the recirculating mariculture system (DF farm), samples DF1, DF2, DF3, DF4, DF5, and DF6 refer to the influent, fish pond, protein separator, biochemical pool for wastewater treatment, biological aerobic pool for wastewater advanced treatment, and the recycled water. relative abundance of the total ARGs in WWTPs was 27-54 ppm (Yang et al., 2013). Based on Li et al. (2015), the relative abundance of ARGs in river was $4.0 \times 10^{-4}-1.4 \times 10^{-2} \mathrm{ppm}$, in drinking water was $2.9 \times 10^{-3}-1.1 \times 10^{-2} \mathrm{ppm}$, and in the faces and wastewater from livestock farm was $1.8 \times 10^{-2}-7.0 \times 10^{-2} \mathrm{ppm}$. The relative abundance of the total ARGs in long-term greenhouse soils was between 0.1 and $20 \times 10^{4} \mathrm{ppm}$ (Fang et al., 2015). The relative abundance of total ARGs in the industrial mariculture systems was about $0.27-4.55 \times 10^{-4} \mathrm{ppm}$, which is much lower than that of other environments, such as rivers, drinking water, feces and wastewater from livestock farm, manured soils, and WWTPs. However, the contribution of industrial mariculture systems on the ARGs pollution in coastal area could not be neglected. Human activity including mariculture farm has been confirmed to be responsible for the occurrence of ARGs in estuarine sediments (Muziasari et al., 2017; Zhu et al., 2017). In this investigation, the relative abundance of ARGs in effluent samples such as ZZ7 was still very high and had almost the same ARGs types with the adjacent aquaculture farms, suggesting that mariculture farms have some influence on the surrounding coastal waters.

By comparing samples ZZ1 and ZZ2, it was found that the ARGs relating to tetracycline and LSM increased significantly, and those relating to bacitracin reduced (Fig. 3), indicating that tetracycline, lincosamide, streptogramin and macrolide might be used in P. vannamei culturing. The comparison between samples ZZ3 and ZZ1 showed that the resistance genes of tetracycline, sulfonamide and chloramphenicol were significantly increased in sample ZZ3, indicating that tetracycline, sulfonamide, and chloramphenicol were used in $S$. maximus culturing.
The comparison results of samples ZZ4 and ZZ1 showed that the genes conferring resistance to tetracycline, sulfonamide, chloramphenicol and streptomycin increased significantly in sample ZZ4, indicating that the four types of antibiotics were used in fish-fry of $S$. maximus culturing. Comparing to sample ZZ1, ARGs relating to tetracycline, sulfonamide and streptomycin increased obviously in sample ZZ5, which meant that the three types of antibiotics might be used in the P. stellatus breeding. Comparing the samples from DF farm, the relative abundance of erythromycin was the highest in DF2, indicating that erythromycin might be used in A. salmon farming. Over all, tetracycline, sulfonamide, chloramphenicol and streptomycin, lincosamide, streptogramin and macrolide might be used in $\mathrm{ZZ} \mathrm{farm,} \mathrm{and} \mathrm{erythromycin} \mathrm{might} \mathrm{be} \mathrm{used} \mathrm{in}$ DF farm. All of the antibiotics mentioned here were widely used in the aquaculture to inhibit pathogen bacteria (Heuer et al., 2009). Among these chemicals, chloramphenicol and erythromycin have not been used in the investigated fish farms in this study for over 3 years since chloramphenicol and erythromycin have been banned to use in aquaculture because of their biological toxicity to human health according to Guidelines for Drug Use for Non-public Nuisance Food and Fishing (NY5017-2002, China). The detection of the ARGs referring to the two antibiotics in the mariculture indicates that the ARGs might be persistent in the mariculture systems.

In contrast to samples ZZ2, ZZ3, ZZ4 and ZZ5, the relative abundance of the various types of resistance genes was lower in ZZ6, indicating that ARGs were reduced by treatments in stabilization pond in the conventional mariculture systems. The relative abundance of ARGs in sample DF2 were the lowest one (Fig. 3a) in DF farm, which might 


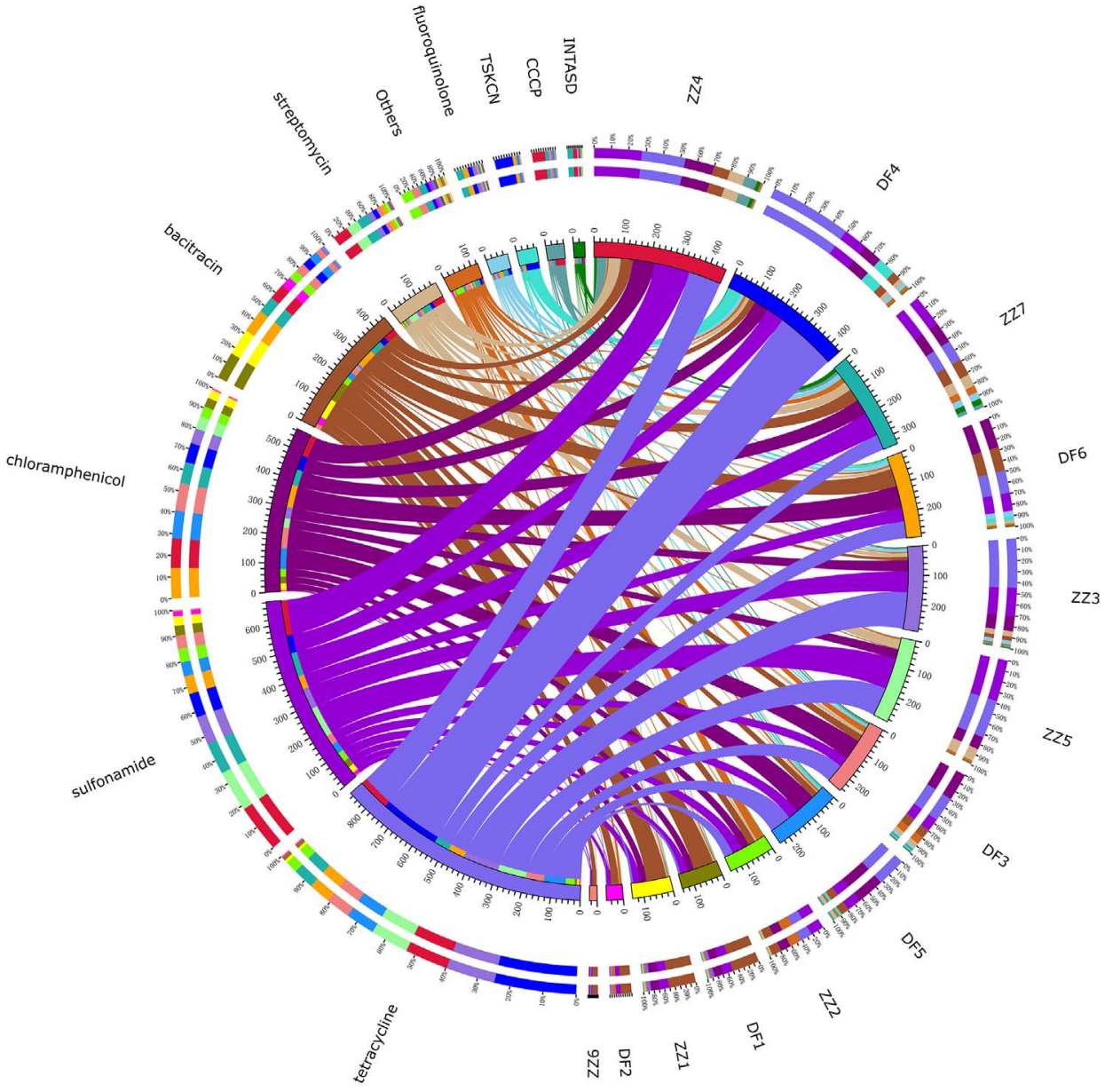

Fig. 2. Distribution of ARG types (in relation to antibiotic types) detected in the 13 samples. The data were visualized using Circos. The outmost two circles list names of samples and ARGs types. The third circle represents the reads number of each ARG types. The bar width of the bars between ARG types and samples correlates to the percentages of respective ARG types in these samples. The different colors in the circle represent different samples and ARG types. In the conventional mariculture system (ZZ farm), samples ZZ1, $\mathrm{ZZ6}$, and ZZ7 refer to the influent, the stabilization and coastal water sample, respectively while ZZ2, ZZ3, $Z Z 4$, and ZZ5 refer to the sample of fish pond 1, 2, 3, and 4 , respectively. In the recirculating mariculture system (DF farm), samples DF1, DF2, DF3, DF4, DF5, and DF6 refer to the influent, fish pond, protein separator, biochemical pool for wastewater treatment, biological aerobic pool for wastewater advanced treatment, and the recycled water. also be caused due to ARGs settlement or adsorption on sediment. Previous studies (Jang et al., 2017; Sui et al., 2017; Zhang et al., 2016) have reported anaerobic digestion reduced the relative abundance of ARGs related to tetracycline. The anaerobic conditions in the sediment of the stabilization pond might also lead to the decrease in the ARGs. The relative abundance of ARGs in sample ZZ7 collected from the adjacent coastal was higher than that of sample ZZ6, which was because the wastewater of other mariculture farms was also discharged in this area. The ARGs types and the proportion of each type were different in different water operation units of the recirculating systems. In the case of the samples collected from the recirculating system (DF farm), the relative abundance of ARGs in samples DF3, DF4 and DF5 was higher than that of samples DF1 and DF2, especially in terms of sample DF4 with the highest ARGs relative abundance (Fig. 3a), indicating that the wastewater treatment systems used in this aquaculture farm has less effect in ARGs reduction. Worst of all, ARGs pollution were accumulated and promoted in wastewater biochemical treatment process. Many investigations on the ARGs distribution in WWTPs reported that the abundance in effluents and activated sludge were very high and the common treatment methods of WWTPs had low removal effect on ARGs (Guo et al., 2017; LaPara et al., 2011; Munir et al., 2011). In this study, the ARGs relative abundance of the recycled water sample in the recirculating system (DF6) was relatively high, indicating the low ARGs removal efficiency of the water treatment processes in coastal industrial mariculture sytems. Compared with samples DF4, DF1 and DF3, the relative abundance of ARGs reference to tetracycline and TSKCN was significantly increased in sample DF4, indicating that these ARGs were accumulated in biochemical processing. Compared with samples DF5 and DF4, the relative abundance of ARGs relating to tetracycline and TSKCN was significantly reduced in DF5, indicating that the ARGs were carried with the active organisms in the biochemical processing, which was consistent with the foregoing result. Compared with samples DF6 and DF1, the relative abundance of all ARGs in DF6 was higher than that of DF1, indicating that the recirculation unit might lead to the accumulation of ARGs in the aquaculture system. The results showed that ARGs could be accumulated in typical mariculture systems including the conventional (without recirculation unit) and recirculating mariculture systems. Compared with samples DF6 and DF2, the relative abundance of all kinds of ARGs increased in sample DF6, indicating that ARGs accumulated in the water treatment systems could enter into the fish pond through water recycling. It is safe to argue that the recirculating mariculture system could effectively reduce the ARGs emissions into the coastal environments because of the low wastewater discharge.

\subsection{Correlation between ARGs and microbial community}

The occurrence of ARGs is correlated with the structure and composition of the bacterial community (Guo et al., 2017). Bacteria harboring different ARGs may amplify or attenuate in different environmental conditions and consequentially change the profiles of ARGs in different samples. Metagenomic approach can examine not only the broad-spectrum profile of ARGs but also the composition of microbial communities at the same time so that the correlation of bacteria community and ARGs profiles can be investigated. Proteobacteria and Bacteroidetes were the two dominant phyla in antibiotic resistance bacteria among the samples collected from ZZ farm, with the percentages of $36 \%$ and $9 \%$, respectively (Fig. 5a). Especially for Proteobacteria, the proportion of it in the antibiotic resistance bacteria was over than the proportion in the total microbial community, indicating 

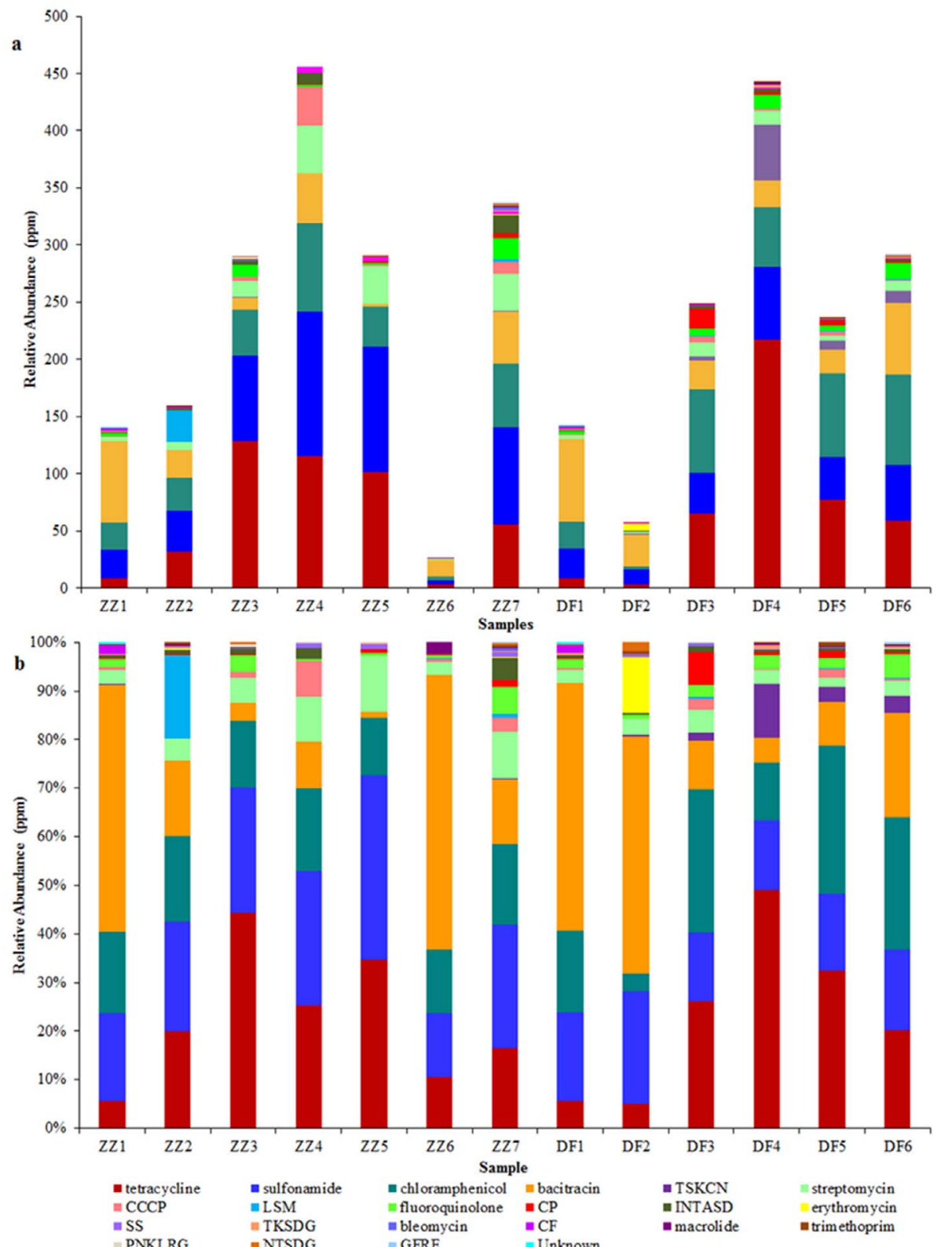

Fig. 3. The relative abundance bar of antibiotic resistance genes found in these samples. (a) The relative abundance (ppm) of ARGs in each samples, and the data in the graph is $10^{6}$ times of the original relative abundance. (b) The percentages of each type of AGRs in the total ARGs found in these samples. The genes can resist two or more antibiotics are described in acronyms: tigecycline-streptomycin-kanamycin-ciprofloxacin-norfloxacin (TSKCN), cephamycin-cephalosporin-carbapenem-penicillin (CCCP), lincosamidestreptogramin-macrolide (LSM), carbenicillin-penicillin (CP), isepamicin-netilmicin-tobramycin-amikacin-sisomicin-dibekacin ((INTASD), spectinomycin-streptomycin (SS), tobramycin-kanamycin-sisomicin-dibekacin-gentamicin (TKSDG), chloramphenicol-fluoroquinolone (CF), paromomycin-neomycin-kanamycin-lividomycin-ribostamycin-gentamincin (NTSDG), glycylcycline-fluoroquinolone-roxithromycin-erythromycin (GFRE). Samples ZZ1, ZZ6, and ZZ7 refer to the influent, the stabilization and coastal water sample in conventional mariculture system, respectively while ZZ2, ZZ3, ZZ4, and ZZ5 refer to the sample of fish ponds $1,2,3$, and 4, respectively. Samples DF1, DF2, DF3, DF4, DF5, and DF6 refer to the influent, fish pond, protein separator, biochemical pool for wastewater treatment, biological aerobic pool for wastewater advanced treatment, and the recycled water in the recirculating system. that Proteobacteria carrying with more resistance genes than other phylum. Proteobacteria and Bacteroidetes were also the dominant phyla in antibiotic resistance bacteria among the samples collected from $\mathrm{DF}$ farm, with the percentages of $36 \%$ and $4 \%$, respectively (Fig. 5b), indicating that Proteobacteria carrying with more resistance genes than other phylum. Additionally, the bacteria of Actinobacteria, Acidobacteria, and Cyanobacteria in both mariculture systems also carried with some resistance genes. It must be pointed out that typical nitrifying bacteria such as Nitrospinae in the recirculating systems also carried with some resistance genes.

Comparing the metagenomics analysis results of the two farms, it was found that the most dominant phyla were Proteobacteria and Bacteroidetes, and the main ARGs were genes conferring resistance to tetracycline, sulfonamide, chloramphenicol and bacitracin in both of the two industrial mariculture farms. The proportion of Proteobacteria in the antibiotic resistance bacteria was over than the proportion in the total microbial community in both mariculture systems, indicating that Proteobacteria carrying with more resistance genes than other phylum. Xiong et al. (2015) also found the dominant phyla were Proteobacteria, Bacteroidetes and Firmicutes in sediment samples and Proteobacteria,
Actinobacteria and Bacteroidetes in water samples from aquaculture environment in Guangdong, China. Colombo et al. (2016) found Proteobacteria was the dominant phylum in aquaculture facility. Previous studies (Gao et al., 2012; Muziasari et al., 2017; Su et al., 2011; Xiong et al., 2015) on the ARGs in aquaculture farms also indicated that the dominant ARGs types also were tetracycline and sulfonamide.

\subsection{Antibiotic resistance mechanisms of the ARGs in coastal industrial mariculture systems}

Understanding the antibiotic resistance mechanisms of the ARGs prevalent in mariculture environment is important to assess the potential risk on public health.

Six main antibiotic resistance mechanisms of the ARGs have been introduced in ARDB database (http://ardb.cbcb.umd.edu/browsegene. shtml), and these mechanisms are closely associated with microbial metabolic activity (Miranda et al., 2013).

Based on the antibiotic resistance mechanisms of the detected ARGs and the taxa annotations, the distribution of antibiotic resistance mechanisms in different phyla was shown in Fig. 6. The antibiotic 


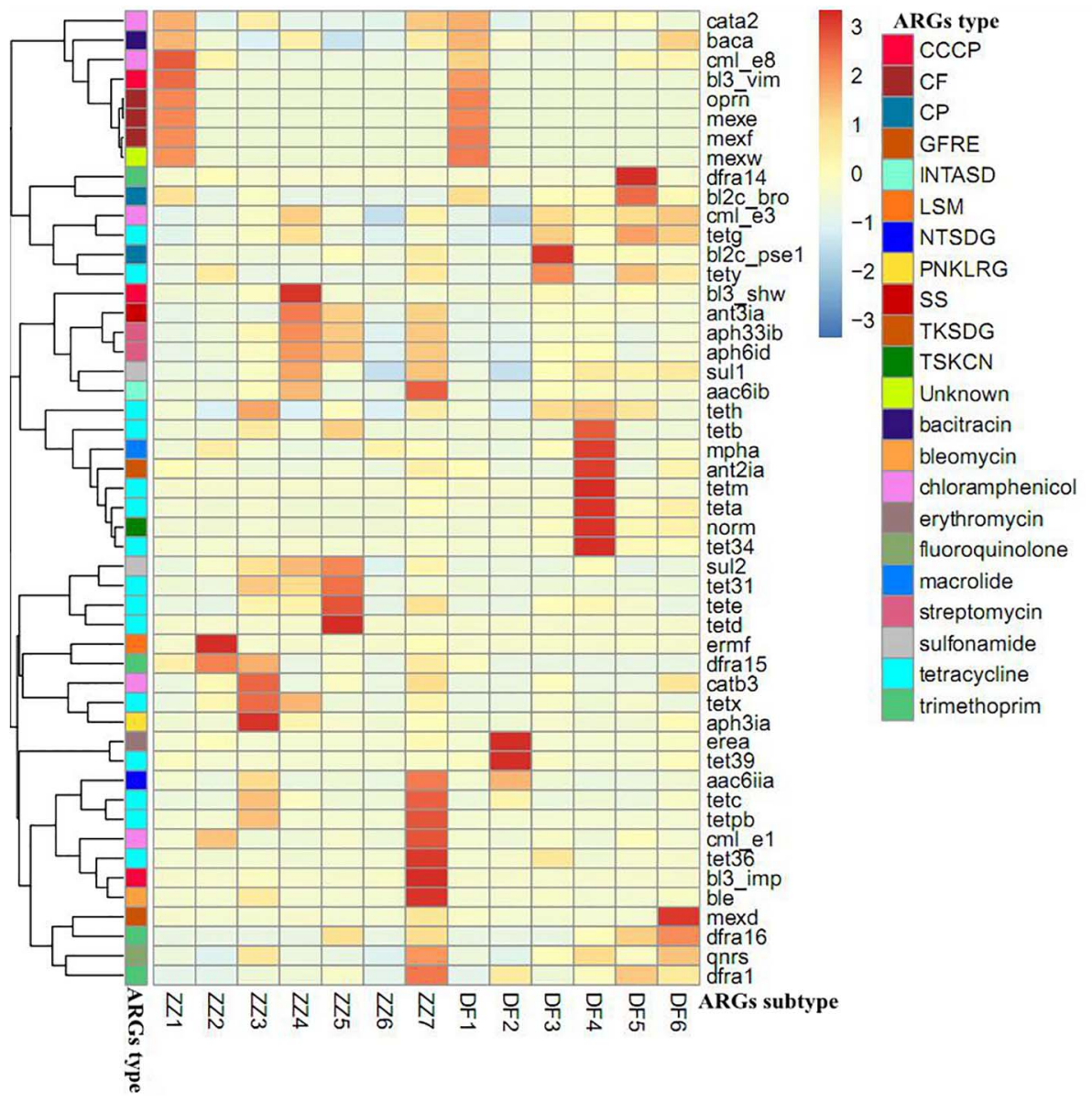

Fig. 4. Heat map of relative abundance of ARGs subtype in coastal industrial mariculture systems. The relative abundance was standardized as Z-value. The genes that can resist two or more antibiotics are described in acronyms: tigecycline-streptomycin-kanamycin-ciprofloxacin-norfloxacin (TSKCN), cephamycin-cephalosporin-carbapenem-penicillin (CCCP), lincosamide-streptogramin-macrolide (LSM), carbenicillin-penicillin (CP), isepamicin-netilmicin-tobramycin-amikacin-sisomicin-dibekacin ((INTASD), spectinomycin-streptomycin (SS), tobramycin-kanamycin-sisomicin-dibekacin-gentamicin (TKSDG), chloramphenicolfluoroquinolone (CF), paromomycin-neomycin-kanamycinlividomycin-ribostamycin-gentamincin (NTSDG), glycylcycline-fluoroquinolone-roxithromycin-erythromycin (GFRE). In the conventional mariculture system (ZZ farm), samples $Z Z 1, Z Z 6$, and ZZ7 refer to the influent, the stabilization and coastal water sample, respectively while ZZ2, ZZ3, ZZ4, and ZZ5 refer to the sample of fish pond 1, 2, 3, and 4, respectively. In the recirculating mariculture system (DF farm), samples DF1, DF2, DF3, DF4, DF5, and DF6 refer to the influent, fish pond, protein separator, biochemical pool for wastewater treatment, biological aerobic pool for wastewater advanced treatment, and the recycled water. a

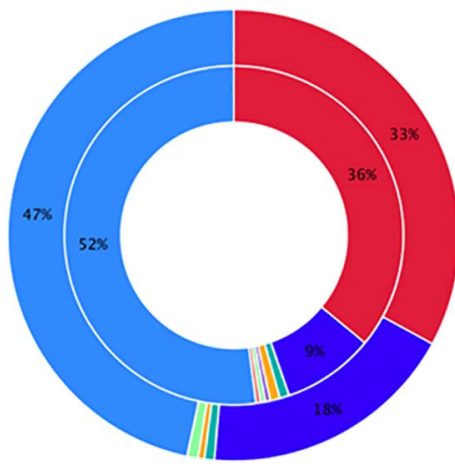

- Proteobacteria

- Bacteroidetes

- Actinobacteria

- Cyanobacteria

- Acidobacteria

Verrucomicrobia

- Fusobacteria

- Others

b

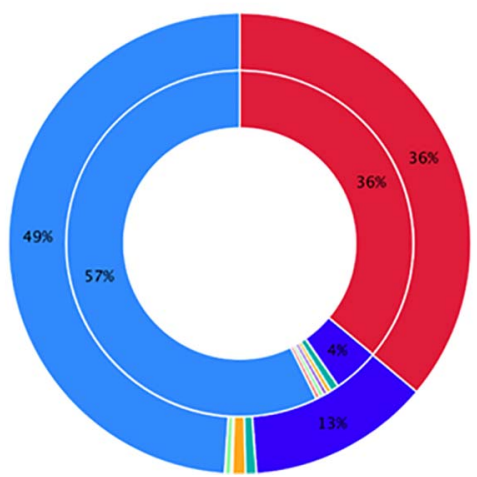

- Proteobacteria

- Bacteroidetes

Actinobacteria

Planctomycetes

- Acidobacteria

- Cyanobacteria

- Nitrospinae

- Others

Fig. 5. The attribution analysis of ARGs and bacteria species in the conventional (a) and recirculating (b) mariculture systems showed by circle map. The inner circle is the distribution of bacteria species of the total ARGs, and the outer circle is the species distribution of all samples. resistance mechanisms of the ARGs detected in the testing samples included efflux proteins, $\beta$-lactamase class $\mathrm{B}, \beta$-lactamase class A, ribosomal protection proteins, acetyltransferases, phosphotransferases, nucleotidyltransferases, MFS transporters, RND transporters, erm_class_rRNA_methylases, phosphorylases, and esterases. In the case of the antibiotic resistance mechanisms in the predominant bacteria in mariculture systems, the antibiotic resistance mechanisms of the ARGs in Proteobacteria were $\beta$-lactamases (49\%), acetyltransferases (18\%), and nucleotidyltransferases $(11 \%)$ while those in Bacteroidetes were ribosomal protection proteins. The $\beta$-lactamases could provide multiresistance to $\beta$-lactam antibiotics such as penicillins, cephalosporins, cephamycins, and carbapenems by breaking these antibiotics' structure (Docquier and Mangani, 2018). Ribosomal protection proteins were considered as the mechanism of tetracycline resistance (Li et al., 2013). The acetyltransferases, nucleotidyltransferases, and phosphotransferases had been identified as the aminoglycosides and chloramphenicol resistance mechanisms (del Campo et al., 2000; Hummel et al., 2007). The efflux proteins and multidrug transporters (MFS transporters and RND transporters) had been identified as the mechanisms for the multidrug resistance (Du et al., 2015; Yilmaz and Özcengiz, 2017).

\section{Conclusions}

The ARGs profiles of the coastal industrial mariculture systems were firstly investigated through metagenomics approach. Fifty ARGs subtypes belonging to 21 types were detected, indicting the wide-spectrum profiles of ARGs. The most predominant ARGs were genes conferring resistance to tetracycline, sulfonamide, chloramphenicol and bacitracin. The antibiotic resistance mechanisms of the ARGs in dominant bacteria were the $\beta$-lactamase and ribosomal protection protein. The 


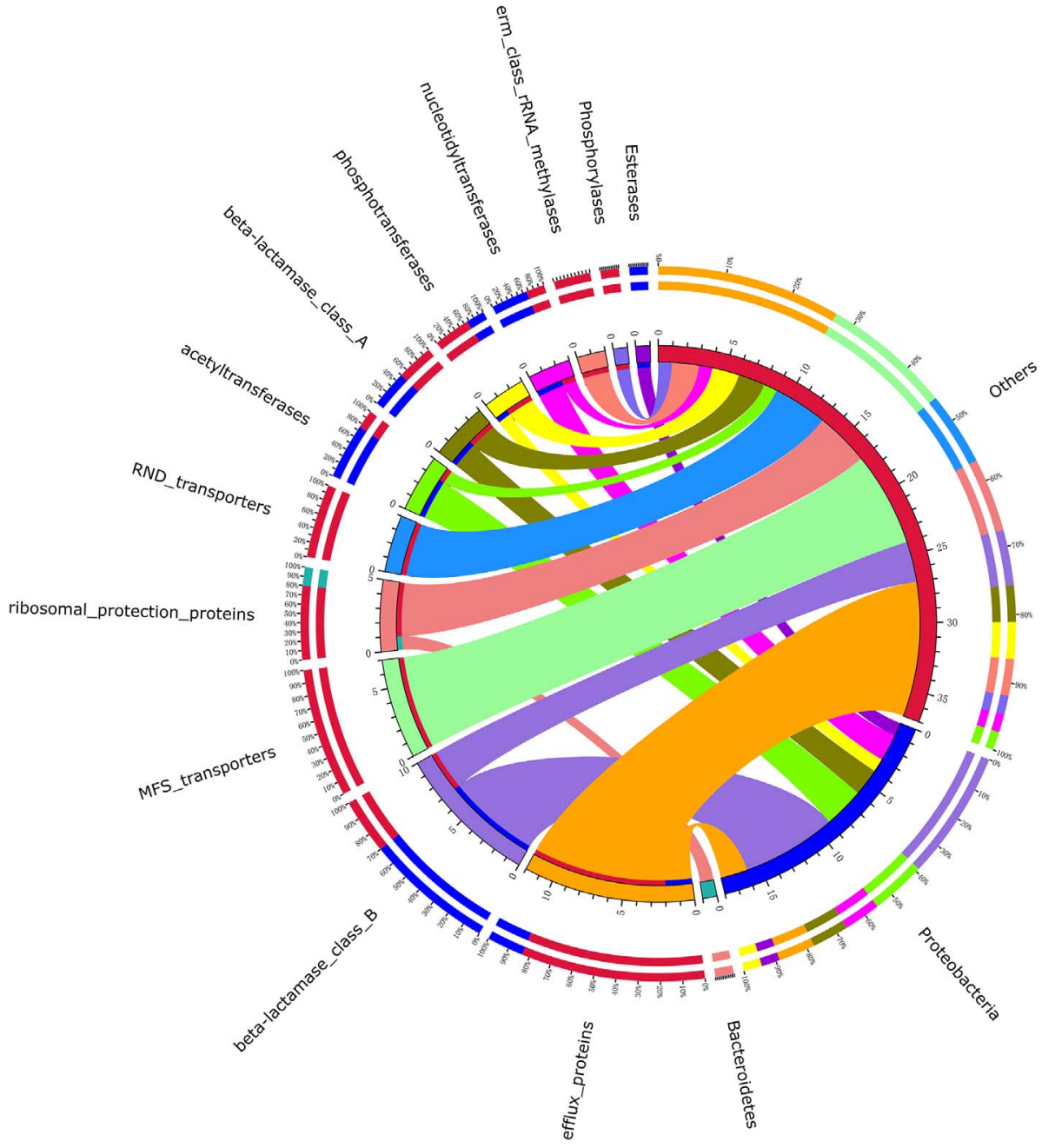

Fig. 6. The relationships between antibiotic resistance mechanisms and bacteria phyla. The outmost two circles list names of the resistance mechanisms and bacteria phyla. The third circle represents the reads number of ARGs in this mechanism. The bar width of the bars between resistance mechanisms and bacteria phyla correlates to the percentages of respective mechanism in these phyla. The different colors in the circle represent different resistance mechanisms and bacteria phyla.

investigation showed that coastal industrial mariculture systems were important ARGs reservoirs, indicating that recirculating systems are the promising mariculture systems in the terms of ARGs pollution control due to the low wastewater discharge.

\section{Acknowledgements}

This work was financially supported by National Natural Science Foundation of China (No. 41671319), One Hundred-Talent Plan of Chinese Academy of Sciences (Grant numbers of Y629041021 and Y610061033), Two-Hundred Talents Plan of Yantai (Y739011021), and Research Program of CAS Key Laboratory of Coastal Environmental Processes and Ecological Remediation (No. 1189010002).

\section{References}

Abriouel, H., Omar, N.B., Molinos, A.C., López, R.L., Grande, M.J., Martínez-Viedma, P., Ortega, E., Cañamero, M.M., Galvez, A., 2008. Comparative analysis of genetic diversity and incidence of virulence factors and antibiotic resistance among enterococcal populations from raw fruit and vegetable foods, water and soil, and clinical samples. Int. J. Food Microbiol. 123, 38-49.

Aydin, S., Ince, B., Ince, O., 2016. Assessment of anaerobic bacterial diversity and its effects on anaerobic system stability and the occurrence of antibiotic resistance genes. Bioresour. Technol. 207, 332-338.

Buchfink, B., Xie, C., Huson, D.H., 2015. Fast and sensitive protein alignment using DIAMOND. Nat. Methods 12, 59-60.

Campo, R., Tenorio, C., Rubio, C., Castillo, J., Torres, C., Gómez-Lus, R., 2000. Aminoglycoside-modifying enzymes in high-level streptomycin and gentamicin resistant Enterococcus spp. in Spain. Int. J. Antimicrob. Agents 15, 221-226.

Colombo, S., Arioli, S., Guglielmetti, S., Lunelli, F., Mora, D., 2016. Virome-associated antibiotic-resistance genes in an experimental aquaculture facility. FEMS Microbiol.
Ecol. 92 (3) fiw003.

Docquier, J., Mangani, S., 2018. An update on $\beta$-lactamase inhibitor discovery and development. Drug Resist. Update 36, 13-29.

Du, D., van Veen, H.W., Murakami, S., Pos, K.M., Luisi, B.F., 2015. Structure, mechanism and cooperation of bacterial multidrug transporters. Curr. Opin. Struc. Biol. 33, 76-91.

Fang, H., Wang, H., Cai, L., Yu, Y., 2015. Prevalence of antibiotic resistance genes and bacterial pathogens in long-term manured greenhouse soils as revealed by metagenomic survey. Environ. Sci. Technol. 49, 1095-1104.

Gao, P., Mao, D., Luo, Y., Wang, L., Xu, B., Xu, L., 2012. Occurrence of sulfonamide and tetracycline-resistant bacteria and resistance genes in aquaculture environment. Water Res. 46, 2355-2364.

Guo, J., Li, J., Chen, H., Bond, P., Yuan, Z., 2017. Metagenomic analysis reveals wastewater treatment plants as hotspots of antibiotic resistance genes and mobile genetic elements. Water Res. 123, 468-478.

Heuer, O.E., Kruse, H., Grave, K., Collignon, P., Karunasagar, I., Angulo, F.J., 2009. Human health consequences of use of antimicrobial agents in aquaculture. Clin. Infect. Dis. 49 (8), 1248-1253.

Hu, Y., Yang, X., Qin, J., Lu, N., Cheng, G., Wu, N., Pan, Y., 2013. Metagenome-wide analysis of antibiotic resistance genes in a large cohort of human gut microbiota. Nat. Commun. 4, 2151.

Hummel, A., Holzapfel, W.H., Franz, C.M., 2007. Characterisation and transfer of antibiotic resistance genes from enterococci isolated from food. Syst. Appl. Microbiol. $30,1-7$.

Jang, H.M., Shin, J., Choi, S., Shin, S.G., Park, K.Y., Cho, J., Kim, Y.M., 2017. Fate of antibiotic resistance genes in mesophilic and thermophilic anaerobic digestion of chemically enhanced primary treatment (CEPT) sludge. Bioresour. Technol. 244, 433-444.

Karlsson, F.H., Fâk, F., Nookaew, I., Tremaroli, V., Fagerberg, B., Petranovic, D., Bäckhed, F., Nielsen, J., 2012. Symptomatic atherosclerosis is associated with an altered gut metagenome. Nat. Commun. 3, 1245.

Kristiansson, E., Fick, J., Janzon, A., Grabic, R., Rutgersson, C., Weijdegard, B., Soderstrom, H., Larsson, D.G.J., 2011. Pyrosequencing of antibiotic-contaminated river sediments reveals high levels of resistance and gene transfer elements. PLoS One 6 (2), e17038.

Krzywinski, M., Schein, J., Birol, I., Connors, J., Gascoyne, R., Horsman, D., Jones, S.J., 
Marra, M.A., 2009. Circos: an information aesthetic for comparative genomics. Genome Res. 19 (9), 1639-1645.

LaPara, T.M., Burch, T.R., McNamara, P.J., Tan, D.T., Yan, M., Eichmiller, J.J., 2011. Tertiary-treated municipal wastewater is a significant point source of antibiotic resistance genes into Duluth-superior Harbor. Environ. Sci. Technol. 45 (22), 9543-9549.

Li, W., Atkinson, G.C., Thakor, N.S., Allas, Ü., Lu, C., Chan, K., Tenson, T., Schulten, K., Wilson, K.K., Hauryliuk, V., Frank, J., 2013. Mechanism of tetracycline resistance by ribosomal protection protein Tet(O). Nat. Commun. 4, 1477.

Li, J., Jia, H., Cai, X., Zhong, H., Feng, Q., Sunagawa, S., Arumugam, M., 2014. An integrated catalog of reference genes in the human gut microbiome. Nat. Biotechnol. 32 (8), 834-841.

Li, B., Yang, Y., Ma, L., Ju, F., Guo, F., Tiedje, J.M., Zhang, T., 2015. Metagenomic and network analysis reveal wide distribution and co-occurrence of environmental antibiotic resistance genes. ISME J. 9 (11), 2490-2502.

Miranda, C.D., Tello, A., Keen, P.L., 2013. Mechanisms of antimicrobial resistance in finfish aquaculture environments. Front. Microbiol. 4, 233.

Munir, M., Wong, K., Xagoraraki, I., 2011. Release of antibiotic resistant bacteria and genes in the effluent and biosolids of five wastewater utilities in Michigan. Water Res. 45 (2), 681-693.

Muziasari, W.I., Pitkänen, L.K., Sørum, H., Stedtfeld, R.D., Tiedje, J.M., Virta, M., 2017. The resistome of farmed fish feces contributes to the enrichment of antibiotic resistance genes in sediments below Baltic Sea fish farms. Front. Microbiol. 7, 2137.

Pruden, A., Pei, R., Storteboom, H., Carlson, K.H., 2006. Antibiotic resistance genes as emerging contaminants: studies in northern Colorado. Environ. Sci. Technol. 40, 7445-7450.

Qin, N., Yang, F., Li, A., Prifti, E., Chen, Y., Shao, L., Guo, J., 2014. Alteration of the human gut microbiome in liver cirrhosis. Nature 513 (7516), 59-64.

Sapkota, A., Sapkota, A.R., KucharskiM, Burke J, McKenzie, S., Walker, P., Lawrence, R., 2008. Aquaculture practices and potential human health risks: current knowledge and future priorities. Environ. Int. 34, 1215-1226.

Su, H.C., Ying, G.G., Tao, R., Zhang, R.Q., Fogarty, L.R., Kolpin, D.W., 2011. Occurrence of antibiotic resistance and characterization of resistance genes and integrons in Enterobacteriaceae isolated from integrated fish farms in south China. J. Environ.
Monit. 13, 3229-3236.

Sui, Q., Zhang, J., Tong, J., Chen, M., Wei, Y., 2017. Seasonal variation and removal efficiency of antibiotic resistance genes during wastewater treatment of swine farms. Environ. Sci. Pollut. Res. 24 (10), 9048-9057.

Tian, Z., Zhang, Y., Yu, B., Yang, M., 2016. Changes of resistome, mobilome and potential hosts of antibiotic resistance genes during the transformation of anaerobic digestion from mesophilic to thermophilic. Water Res. 98, 261-269.

Villar, E., Farrant, G.K., Follows, M., Garczarek, L., Speich, S., Audic, S., Bittner, L., 2015. Environmental characteristics of Agulhas rings affect interocean plankton transport. Science 348 (6237), 1261447.

Xiao, K.Q., Li, B., Ma, L., Bao, P., Zhou, X., Zhang, T., Zhu, Y.G., 2016. Metagenomic profiles of antibiotic resistance genes (ARGs) in paddy soils from South China. FEMS Microbiol. Ecol. 92 (3) fiw023.

Xiong, W., Sun, Y., Zhang, T., Ding, X., Li, Y., Wang, M., Zeng, Z., 2015. Antibiotics, antibiotic resistance genes, and bacterial community composition in fresh water aquaculture environment in China. Microb. Ecol. 70, 425-432.

Xu, J., Xu, Y., Wang, H., Guo, C., Qiu, H., He, Y., Zhang, Y., Li, X., Meng, W., 2015. Occurrence of antibiotics and antibiotic resistance genes in a sewage treatment plant and its effluent-receiving river. Chemosphere 119, 1379-1385.

Yang, Y., Li, B., Ju, F., Zhang, T., 2013. Exploring variation of antibiotic resistance genes in activated sludge over a four-year period through a metagenomic approach. Environ. Sci. Technol. 47 (18), 10197-10205.

Yang, Y., Li, B., Zou, S., Fang, H.H.P., Zhang, T., 2014. Fate of antibiotic resistance genes in sewage treatment plant revealed by metagenomic approach. Water Res. 62 , 97-106.

Yilmaz, C., Özcengiz, G., 2017. Antibiotics: Pharmacokinetics, toxicity, resistance and multidrug efflux pumps. Biochem. Pharmacol. 133, 43-62.

Zhang, J., Chen, M., Sui, Q., Wang, R., Tong, J., Wei, Y., 2016. Fate of antibiotic resistance genes and its drivers during anaerobic co-digestion of food waste and sewage sludge based on microwave pretreatment. Bioresour. Technol. 217, 28-36.

Zhu, Y.G., Zhao, Y., Li, B., Huang, C.L., Zhang, S.Y., Yu, S., Chen, Y.Y., Zhang, T., 2017. Continental-scale pollution of estuaries with antibiotic resistance genes. Nat. Microbiol. 2, 16270. 